\title{
FOREST DECOMPOSITIONS OF GRAPHS WITH CYCLOMATIC NUMBER 3
}

\author{
E.J. FARRELL \\ Department of Mathematics \\ The University of the West Indies \\ St. Augustine, Trinidad \\ (Received June 15, 1982)
}

ABSTRACT. The simple tree polynomials of the basic graphs with cyclomatic number 3 are derived. From these results, explicit formulae for the number of decompositions of the graphs into forests with specified cardinalities are extracted. Explicit expressions are also given for the number of spanning forests and spanning trees in the graphs. These results complement the results given in [1].

KEY WORDS AND PHRASES. Tree polynomial, simple tree polynomial, forest decomposition, basic graph, cyclomatic number, chain, circuit, graphs with trees attached.

1980 AMS SUBJECT CLASSIFICATION CODES: 05C05, $05 A 10$.

1. INTRODUCTION.

The graphs considered here will be finite and without loops. Let G be such a graph. With every tree $\alpha$ in $G$, let us associate an indeterminate $w_{\alpha}$ called the weight of $\alpha$. With every spanning forest $F$ in $G$, let us associate the weight

$$
w(F)=\prod_{\alpha} w_{\alpha}
$$

where the product is taken over all the elements in F. Then the tree polynomial of $G$ is $\sum w(F)$, where the summation is taken over all the spanning forests in $G$. If we give each tree with $n$ nodes a weight $w_{n}$, then the tree polynomial of $G$, denoted by $T(G ; \underline{w})$, will be a polynomial in the variables $w_{1}, w_{2}, w_{3}$, etc., where $w_{-}=\left(w_{1}, w_{2}, w_{3}, \ldots\right)$. If we put $w_{1}=w$ for all $i$, then the resulting polynomial, denoted by $T(G ; w)$, will be called the simple tree polynomial of $G$. The basic properties of tree polynomials are given in Farre11 [2]. 
By a forest decomposition of $G$, we mean a node disjoint collection of trees in $G$, which span G. Therefore, a forest decomposition is simply a spanning forest. We will use the word decomposition to mean forest decomposition unless otherwise stated. Let $\mathrm{T}$ be a tree. We attach $\mathrm{T}$ to a graph $\mathrm{G}$ by identifying an endnode of $\mathrm{T}$ with a node of G. The basic graphs with cyclomatic number 3 is the minimum set of graphs with cyclomatic number 3, with the property that any other graph with cyclomatic number 3 can be obtained from one of these graphs by attaching trees to it.

We will derive the simple tree polynomials of the basic graphs with cyclomatic number 3. From these polynomials, we will deduce results about (i) forest decompositions and (ii) spanning trees, in graphs with cyclomatic number 3 . We refer the reader to Harary [4] for the basic definitions in Graph Theory.

2. SOME FUNDAMENTAL RESULTS.

Let $x y$ be an edge of a graph $G$. By partitioning the spanning covers of $G$ into two classes (i) those containing $x y$ and (ii) those not containing $x y$, we obtain the following theorem.

THEOREM 1. Let $G$ be a graph containing an edge $x y$. Let $G^{\prime}$ be the graph obtained from $G$ by deleting $x y$, and $G *$ the graph obtained from $G$ by identifying nodes $x$ and $y$ (and omitting any loops formed). Then

$$
T(G ; w)=T\left(G^{\prime} ; w\right)=T(G * ; w)
$$

The algorithm implied by this theorem will be called the reduction process.

Let $G$ be a graph consisting of two blocks $A$ and $B$ with a common cutnode $x$. Let $F_{1}$ and $F_{2}$ be spanning forests in $A$ and $B$ respectively. Let $\alpha_{1}$ and $\alpha_{2}$ be the elements of $F_{1}$ and $F_{2}$ respectively containing node $x$, and $\alpha$ the tree obtained by attaching $\alpha_{1}$ to $\alpha_{2}$ using node $x$. Then $\left(F_{1}-\left\{\alpha_{1}\right\} \cup\left(F_{2}-\left\{\alpha_{2}\right\}\right) \cup\{\alpha\}\right.$ will be a spanning forest in $G$, with cardinality $\left|F_{1}\right|+\left|F_{2}\right|-1$. Hence, a spanning forest in A could be "combined" with a spanning forest in B to yield a spanning forest in $G$, with one less component. This observation leads to the following results.

LEMMA 1. Let $G$ be a graph consisting of two blocks $A$ and $B$. Then

$$
T(G ; w)=w^{-1} T(A ; w) T(B ; w)
$$

THEOREM 2. (The Cutnode Theorem) Let $G$ be a graph consisting of blocks $\mathrm{B}_{1}, \mathrm{~B}_{2}, \ldots, \mathrm{B}_{\mathrm{n}} \cdot$ Then 


$$
T(G ; w)=w^{-(n-1)} \prod_{i=1}^{n} T\left(B_{i} ; w\right)
$$

The following theorem can be easily proved.

THEOREM 3. (The Component Theorem) Let G be a graph consisting of components $\mathrm{H}_{1}, \mathrm{H}_{2}, \ldots, \mathrm{H}_{\mathrm{n}}$. Then $\mathrm{T}(\mathrm{G} ; \mathrm{w})=\prod_{i=1}^{\mathrm{n}} \mathrm{T}\left(\mathrm{H}_{\mathrm{i}} ; \mathrm{w}\right)$.

\section{PRELIMINARY RESULTS.}

It is clear that the removal of any $k-1$ edges from a tree $T_{p}$ with $p$ nodes $(k<p-1)$ yields a spanning forest with $\mathrm{k}$ components. Hence we have

LEMMA 1.

$$
T\left(T_{p} ; w\right)=w(1+w)^{p-1}
$$

LEMMA 2.

$$
\mathrm{T}\left(\mathrm{C}_{\mathrm{p}} ; \mathrm{w}\right)=(1+\mathrm{w})^{\mathrm{p}}-1
$$

where $C_{p}$ is the circuit with $p$ nodes.

Most of our results will be given in terms of tree polynomials of circuits. We will therefore use $\phi(p)$ for $T\left(C_{p} ; w\right)$. It can be easily confirmed that $\phi$ has the property

PROPERTY 1.

$$
\phi(m+n)=\phi(m) \phi(n)+\phi(m)+\phi(n)
$$

This property of $\phi$ will be useful in obtaining our results in forms which display the symmetries in the graphs.

Let us denote by $\mathrm{H}_{\mathrm{n}}$ the graph obtained by attaching a tree with n nodes to a nonempty graph G. By using the Cutnode Theorem, we obtain the following lemma

LEMMA 3.

$$
T\left(H_{n} ; w\right)=(1+w)^{n-1} T(G ; w)
$$

By a chain, we will mean a tree with nodes of valencies 1 and 2 only. We add a chain (of length greater than 1) to a graph $G$ (with at least 2 nodes) by identifying the end nodes of the chain with two different nodes of $G$. We will denote by $J_{n}$ the graph obtained by adding a chain with $n$ nodes (denoted by $\mathrm{P}_{\mathrm{n}}$ ) to a graph $\mathrm{G}$.

THEOREM 4.

$$
T\left(J_{n} ; w\right)=w^{-1} \phi(n-1) T(G ; w)+T(G * ; w),
$$


where $G^{*}$ is obtained from $G$ by identifying the nodes common to $G$ and $P_{n}$.

PROOF. Let $x$ and $y$ be the nodes of $J_{n}$ which are common to $G$ and $P_{n}$. Apply the reduction process to $J_{n}$ by deleting an edge of $P_{n}$ which is incident to node $x$. This yields

$$
T\left(J_{n} ; w\right)=T\left(H_{n-1} ; w\right)+T\left(J_{n-1} ; w\right)
$$

This recurrence relation yields

$$
\mathrm{T}\left(\mathrm{J}_{\mathrm{n}} ; \mathrm{w}\right)=\sum_{\mathrm{k}=1}^{\mathrm{n}-2} \mathrm{~T}\left(\mathrm{H}_{\mathrm{n}-\mathrm{k}} ; \mathrm{w}\right)+\mathrm{T}(\mathrm{G} ; \mathrm{w})+\mathrm{T}\left(\mathrm{G}^{*} ; \mathrm{w}\right),
$$

where $G^{*}$ is the graph obtained from $G$ by identifying nodes $x$ and $y$. The result then follows by using Lemma 3 .

The basic graphs with cyclomatic number 2 are shown below. The number of edges in the subgraphs are indicated by $p, q$ and $r$, where $p, q, r>1$.

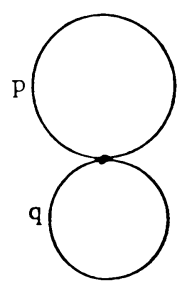

(a)

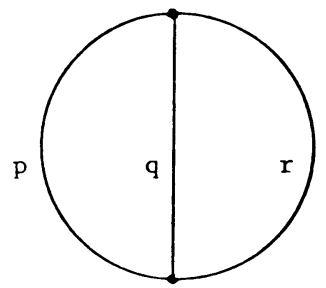

(b)

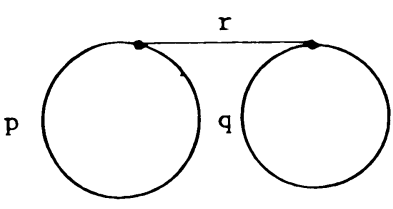

(c)

Figure 1

We will denote the graphs shown in Figures $1(a),(b)$, and (c) by $G(p, q), G(p, q, r)$, and $G((p, q), r)$ respectively. The simple tree polynomials of these graphs were derived in Farrell [1]. We will not reproduce their proofs here, but simply quote the results (N.B., In [1], $p, q$, and $r$ were the number of nodes).

LEMMA 4.

(i) $\quad T(G(p, q) ; w)=w^{-1} \phi(p) \phi(q)$,

(ii) $\mathrm{T}(\mathrm{G}(\mathrm{p}, \mathrm{q}, \mathrm{r}) ; \mathrm{w})=\mathrm{w}^{-1}[\phi(\mathrm{p}+\mathrm{q}+\mathrm{r})-\phi(\mathrm{p})-\phi(\mathrm{q})-\phi(\mathrm{r})]$

and

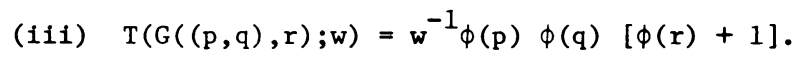

Lemma 4 could be proved by using the Cutnode Theorem for the graphs in (a) and (c), and Theorem 4 for the graph in (b).

4. SIMPLE TREE POLYNOMIALS OF GRAPHS WITH CYCLOIATIC NUMBER 3.

The basic graphs with cyclomatic number 3 are shown below. 


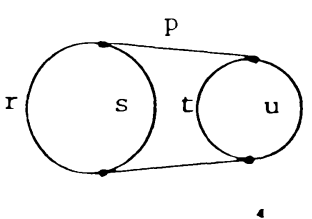

(a)

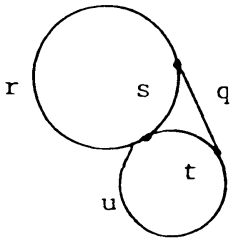

(b)

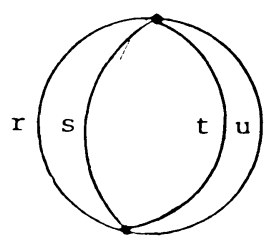

(c)

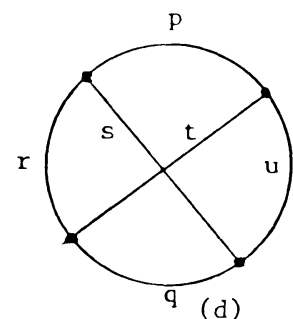

(d)

Figure 2

The lengths of the chains are indicated on the diagrams by the symbols $p, q, r, s, t$, and $u$. We will assume that these lengths are all greater than 1 . The graphs shown in Figures 2(a), (b), (c), and (d) will be represented by $G((r, s),(t, u),(p, q))$, $G((r, s),(t, u), q), G(r, s, t, u)$, and $G((p, q),(r, u),(s, t))$ respectively. We will obtain the polynomials for these graphs in forms which display the symmetries in the graphs. THEOREM 5.

$$
\begin{aligned}
\mathrm{T}(\mathrm{G}(\mathrm{r}, \mathrm{s}, \mathrm{t}, \mathrm{u}) ; \mathrm{w})=\mathrm{w}^{-2}[ & \phi(\mathrm{r}+\mathrm{s}+\mathrm{t}+\mathrm{u})-\phi(\mathrm{r}+\mathrm{s})-\phi(\mathrm{t}+\mathrm{u})-\phi(\mathrm{r}+\mathrm{t}) \\
& -\phi(\mathrm{r}+\mathrm{u})-\phi(\mathrm{s}+\mathrm{t})-\phi(\mathrm{s}+\mathrm{u})+2(\phi(\mathrm{r})+\phi(\mathrm{s}) \\
& +\phi(\mathrm{t})+\phi(\mathrm{u}))] .
\end{aligned}
$$

PROOF. $G(r, s, t, u)$ can be viewed as the graph $G(s, t, u)$ with the chain $P_{r+1}$ added to it. Therefore, Theorem 4 can be immediately applied. In this case, G would be $G(s, t, u)$, and $G *$ would be the graph consisting of three circuits $C_{s}, C_{t}$, and $C_{u}$ all joined to a common node. From Lemma 4,

$$
T(G(s, t, u) ; w)=w^{-1}[\phi(s+t+u)-\phi(s)-\phi(t)-\phi(u)] .
$$

From the Cutnode Theorem, we get

$$
T\left(G^{*} ; w\right)=w^{-2} \phi(s) \phi(t) \phi(u) .
$$

Hence, by substituting in Theorem 4, we get

$$
T(G(r, s, t, u) ; w)=w^{-2}\{\phi(r)[\phi(s+t+u)-\phi(s)-\phi(t)-\phi(u)]+\phi(s) \phi(t) \phi(u)\} .
$$

The symmetric result then follows by using Property 1.

THEOREM 6 .

$$
\begin{aligned}
\mathrm{T}(\mathrm{G}((\mathrm{r}, \mathrm{s}),(\mathrm{t}, \mathrm{u}), \mathrm{q}) ; \mathrm{w})=\mathrm{w}^{-2}[\phi(\mathrm{q}+\mathrm{r}+\mathrm{s}+\mathrm{t}+\mathrm{u})-\phi(\mathrm{q}+\mathrm{r}+\mathrm{s}) \\
-\phi(\mathrm{q}+\mathrm{t}+\mathrm{u})-\phi(\mathrm{r}+\mathrm{t})-\phi(\mathrm{r}+\mathrm{u})-\phi(\mathrm{s}+\mathrm{t}) \\
-\phi(\mathrm{s}+\mathrm{u})+\phi(\mathrm{q})+2(\phi(\mathrm{r})+\phi(\mathrm{s})+\phi(\mathrm{t})+\phi(\mathrm{u}))] .
\end{aligned}
$$


PROOF. We can view $G((r, s),(t, u), q)$ as the graph $G(r+s, t+u)$ with the chain $P_{q+1}$ added to it. Hence, Theorem 4 can be applied. In this case, $G^{*}$ will be $G(r, s, t, u)$. From Lemma 4 (i),

$$
T(G(r+s, t+u), w)=w^{-1} \phi(r+s) \phi(t+u)
$$

Therefore,

$$
T(G((r, s),(t, u), q) ; w)=w^{-2} \phi(q) \phi(r+s) \phi(t+u)+T(G(r, s, t, u) ; w) .
$$

The result then follows by using Theorem 5 and Property 1 .

The graph $G((p, q),(r, s),(t, u))$ can be viewed as $G((r+s, t+u), q)$ with the chain $\mathrm{P}_{\mathrm{p}+1}$ added to it. Therefore, we can apply Theorem 4. In this case, G* will be $G((r, s),(t, u), q)$. Hence,

$$
\begin{aligned}
T(G((p, q),(r, s),(t, u)) ; w)=w^{-1} \phi(p) & T(G((r+s, t+u), q) ; w) \\
& +T(G((r, s),(t, u), q) ; w) .
\end{aligned}
$$

By using Lemma 4 (iii) and Theorem 6, we obtain the following result, after simplifications and using Property 1.

THEOREM 7.

$$
\begin{aligned}
\mathrm{T}(\mathrm{G}(\mathrm{p}, \mathrm{q}),(\mathrm{r}, \mathrm{s}),(\mathrm{t}, \mathrm{u})) ; \mathrm{w})=\mathrm{w}^{-2} & {[\phi(\mathrm{p}+\mathrm{q}+\mathrm{r}+\mathrm{s}+\mathrm{t}+\mathrm{u})-\phi(\mathrm{p}+\mathrm{q}+\mathrm{r}+\mathrm{s})} \\
& -\phi(\mathrm{p}+\mathrm{q}+\mathrm{t}+\mathrm{u})+\phi(\mathrm{p}+\mathrm{q})-\phi(\mathrm{r}+\mathrm{t}) \\
& -\phi(\mathrm{r}+\mathrm{u})-\phi(\mathrm{s}+\mathrm{t})-\phi(\mathrm{s}+\mathrm{u}) \\
& +2(\phi(\mathrm{r})+\phi(\mathrm{s})+\phi(\mathrm{t})+\phi(\mathrm{u}))] .
\end{aligned}
$$

Notice that the "sum" forms, in which Theorem 5, 6, 7, and 8 are given, will facilitate deductions about forest decompositions of the graphs.

5. FOREST DECOMPOSITIONS OF GRAPHS WITH CYCLOMATIC NUMBER 3.

It is clear that the coefficient of $w^{k}$ in $T(G ; w)$ is $T_{k}(G)$, the number of decompositions of the graph $G$ into spanning forests with $k$ trees, 1.e., the number of kdecompositions of $\mathrm{G}$. By extracting the relative coefficients in Theorems 5, 6, 7, and 8, we obtain the following corollaries in which $\left(\begin{array}{l}k \\ r\end{array}\right)=0$, for $k<r$, and $k^{\prime}=k^{+} 2$. COROLLARY 5.1 .

$$
\begin{aligned}
T_{k}(G(r, s, t, u))= & \left(\begin{array}{c}
r+s+t+u \\
k^{\prime}
\end{array}\right)-\left(\begin{array}{c}
r+s \\
k^{\prime}
\end{array}\right)-\left(\begin{array}{c}
t+u \\
k^{\prime}
\end{array}\right)-\left(\begin{array}{c}
r+t \\
k^{\prime}
\end{array}\right) \\
& -\left(\begin{array}{c}
s+t \\
k^{\prime}
\end{array}\right)-\left(\begin{array}{c}
s+u \\
k^{\prime}
\end{array}\right)+2\left[\left(\begin{array}{c}
r \\
k^{\prime}
\end{array}\right)+\left(\begin{array}{c}
s \\
k^{\prime}
\end{array}\right)+\left(\begin{array}{l}
t \\
k^{\prime}
\end{array}\right)+\left(\begin{array}{l}
u \\
k^{\prime}
\end{array}\right)\right] .
\end{aligned}
$$


COROLLARY 6.1 .

$$
\begin{aligned}
T_{k}(G((r, s),(t, u), q))= & \left(\begin{array}{c}
q+r+s+t+u \\
k^{\prime}
\end{array}\right)-\left(\begin{array}{c}
q+r+s \\
k^{\prime}
\end{array}\right)-\left(\begin{array}{c}
q+t+u \\
k^{\prime}
\end{array}\right) \\
& -\left(\begin{array}{c}
r+t \\
k^{\prime}
\end{array}\right)-\left(\begin{array}{c}
r+u \\
k^{\prime}
\end{array}\right)-\left(\begin{array}{c}
s+t \\
k^{\prime}
\end{array}\right)-\left(\begin{array}{c}
s+u \\
k^{\prime}
\end{array}\right) \\
& +\left(\begin{array}{c}
q^{\prime} \\
k^{\prime}
\end{array}\right)+2\left[\left(\begin{array}{l}
r \\
k^{\prime}
\end{array}\right)+\left(\begin{array}{l}
s \\
k^{\prime}
\end{array}\right)+\left(\begin{array}{l}
t \\
k^{\prime}
\end{array}\right)+\left(\begin{array}{l}
u \\
k^{\prime}
\end{array}\right)\right] .
\end{aligned}
$$

COROLLARY 7.1 .

$$
\begin{aligned}
T_{k^{\prime}}(G((p, q),(r, s),(t, u)))= & \left(\begin{array}{c}
p+q+r+s+t+u \\
k^{\prime}
\end{array}\right)-\left(\begin{array}{c}
p+q+r+s \\
k^{\prime}
\end{array}\right) \\
& -\left(\begin{array}{c}
p+q+t+u \\
k^{\prime}
\end{array}\right)+\left(\begin{array}{c}
p+q \\
k^{\prime}
\end{array}\right)-\left(\begin{array}{c}
r+t \\
k^{\prime}
\end{array}\right)-\left(\begin{array}{c}
r+u \\
k^{\prime}
\end{array}\right) \\
& -\left(\begin{array}{c}
s+t \\
k^{\prime}
\end{array}\right)-\left(\begin{array}{c}
s+u \\
k^{\prime}
\end{array}\right)+2\left[\left(\begin{array}{c}
r \\
k^{\prime}
\end{array}\right)+\left(\begin{array}{l}
s \\
k^{\prime}
\end{array}\right)+\left(\begin{array}{l}
t \\
k^{\prime}
\end{array}\right)+\left(\begin{array}{l}
u \\
k^{\prime}
\end{array}\right)\right] .
\end{aligned}
$$

(Notice that Corollaries 5.1 and 6.1 can be obtained from Corollary 7.1 by putting $\mathrm{p}=\mathrm{q}=0$ and $\mathrm{p}=0$ respectively.)

COROLLARY 8.1.

$$
\begin{aligned}
T_{k}(G((p, q),(r, u),(s, t)))= & \left(\begin{array}{c}
p+q+r+s+t \\
k^{\prime}
\end{array}\right)-\left(\begin{array}{c}
p+r+s \\
k^{\prime}
\end{array}\right)-\left(\begin{array}{c}
q+r+t \\
k^{\prime}
\end{array}\right) \\
& -\left(\begin{array}{c}
p+t+u \\
k^{\prime}
\end{array}\right)-\left(\begin{array}{c}
q+s+u \\
k^{\prime}
\end{array}\right)-\left(\begin{array}{c}
p+q \\
k^{\prime}
\end{array}\right)-\left(\begin{array}{c}
r+u \\
k^{\prime}
\end{array}\right) \\
& -\left(\begin{array}{c}
s+t \\
k^{\prime}
\end{array}\right)+2\left[\left(\begin{array}{l}
p \\
k^{\prime}
\end{array}\right)+\left(\begin{array}{l}
q \\
k^{\prime}
\end{array}\right)+\left(\begin{array}{l}
r \\
k^{\prime}
\end{array}\right)+\left(\begin{array}{l}
s \\
k^{\prime}
\end{array}\right)+\left(\begin{array}{l}
t \\
k^{\prime}
\end{array}\right)+\left(\begin{array}{l}
u \\
k^{\prime}
\end{array}\right)\right] .
\end{aligned}
$$

Let us denote, by $\mathrm{N}(G)$, the total number of decompositions of $G$, 1.e., the total number of different spanning forests in $G$. Then $N(G)$ is the sum of the coefficients of $T(G ; w)$. Hence, by putting $w=1$ in Theorem 7, we obtain the following result.

THEOREM 9.

$$
\begin{aligned}
N(G((p, q),(r, s),(t, u)))= & 2^{p+q}\left(2^{r+s}-1\right)\left(2^{t+u}-1\right) \\
& -\left(2^{r}+2^{s}-2\right)\left(2^{t}+2^{u}-2\right) .
\end{aligned}
$$

By putting $p=0$ and $p=q=0$ respectively, in Theorem 9 , we obtain COROLLARY 9.1 .

$N(G((r, s),(t, u), q))=2^{q}\left(2^{r+s}-1\right)\left(2^{t+u}-1\right)-\left(2^{r}+2^{s}-2\right)\left(2^{t}+2^{u}-2\right)$.

COROLLARY 9.2.

$$
N(G(r, s, t, u))=\left(2^{r+s}-1\right)\left(2^{t+u}-1\right)-\left(2^{r}+2^{s}-2\right)\left(2^{t}+2^{u}-2\right) .
$$

The following result is immediate from Theorem 8 .

COROLLARY 8.2.

$$
\begin{aligned}
N(G((p, q),(r, u),(s, t)))= & 2^{p+q}\left(2^{r+s+t+u}-1\right)-2^{p}\left(2^{r+s}+2^{t+u}-2\right)-2^{q}\left(2^{r+t}+2^{s+u}-2\right) \\
& -4\left[\left(2^{s-1}-1\right)\left(2^{t-1}-1\right)+\left(2^{r-1}-1\right)\left(2^{u-1}-z\right)\right]+2 .
\end{aligned}
$$


Let us denote, by $\Gamma(G)$, the number of spanning trees in $G$. Then $\Gamma(G)$ is the coefficient of $w$ in $T(G ; w)$. Hence, we can obtain the following result from Theorem 7 or from Corollary 7.1 (by putting $k^{\prime}=3$ ).

THEOREM 10.

$$
\begin{aligned}
\Gamma(G((p, q),(r, s),(t, u)))= & \left(\begin{array}{c}
p+q+r+s+t+u \\
3
\end{array}\right)-\left(\begin{array}{c}
p+q+r+s \\
3
\end{array}\right)-\left(\begin{array}{c}
p+q+t+u \\
3
\end{array}\right) \\
& +\left(\begin{array}{c}
p+q \\
3
\end{array}\right)-\left(\begin{array}{c}
r+t \\
3
\end{array}\right)-\left(\begin{array}{c}
r+u \\
3
\end{array}\right)-\left(\begin{array}{c}
s+t \\
3
\end{array}\right)-\left(\begin{array}{c}
s+u \\
3
\end{array}\right)+2\left[\left(\begin{array}{l}
r \\
3
\end{array}\right)+\left(\begin{array}{l}
s \\
3
\end{array}\right)+\left(\begin{array}{l}
t \\
3
\end{array}\right)+\left(\begin{array}{l}
u \\
3
\end{array}\right)\right] .
\end{aligned}
$$

Similar expressions for $\Gamma(G((r, s),(t, u), q))$ and $\Gamma(G(r, s, t, u)$ can be obtained from Theorem 10 by putting $p=0$ and $p=q=0$, respectively, in the formula. Explicit linear formulae for the number of spanning trees in the basic graphs with cyclomatic number 3, have already been given in Farrell [3].

6. NON-BASIC GRAPHS WITH CYCLOMATIC NUMBER 3.

Let $G$ be a non-basic graph with cyclomatic number 3 . Then $G$ consists of one of the basic graphs with trees attached to it. The simple tree polynomial of G could therefore be obtained by using Lemma 3 and the appropriate result in Section 4 . Hence our results cover all graphs with cyclomatic number 3 .

Lemma 3 can be used to obtain the following results for $T_{k}\left(H_{n}\right)$ and $N\left(H_{n}\right)$. The proofs are quite straightforward.

LEMMA 4.

$$
T_{k}\left(H_{n}\right)=\sum_{r=0}^{k}\left(\begin{array}{c}
n-1 \\
r
\end{array}\right) T_{k-r}(G)
$$

LEMMA 5.

$$
N\left(H_{n}\right)=2^{n-1} N(G)
$$

Lemma 4 and the results given in Section 5 can be used to find the number of kdecompositions of non-basic graphs with cyclomatic number 3 . Similarly, Lemma 5, together with Theorem 9, can be used to find the number of spanning forests in non-basic graphs with cyclomatic number 3 .

7. DISCUSSION.

We are aware that some of the results given in Section 5 and 6 can be deduced by straightforward combinatorial arguments. However, they not only provide useful checks for some of the major results, but also serve to illustrate a new powerful technique afforded by the tree polynomial. 


\section{REFERENCES}

1. FARRELL, E.J. Forest Decompositions of Graphs with Cyclomatic Number 2, submitted to the International Journal of Mathematics and Mathematical Sciences.

2. FARRELL, E.J. The Tree Polynomial of a Graph, Journal of Combinatorics, Information and System Sciences 4 (3) (1979), $211-218$.

3. FARRELL, E.J. Spanning Trees in Graphs with Cyclomatic Numbers 2, 3, and 4, J. of Combinatorics, Information and System Sciences, to appear.

4. HARARY, F. Graph Theory, Addison-Wesley, Reading, Mass., 1969. 


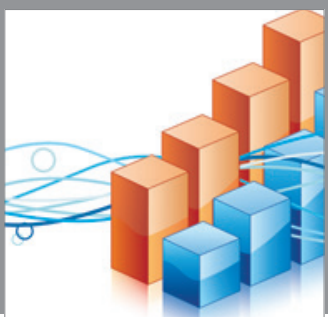

Advances in

Operations Research

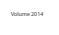

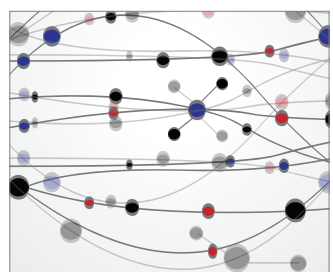

\section{The Scientific} World Journal
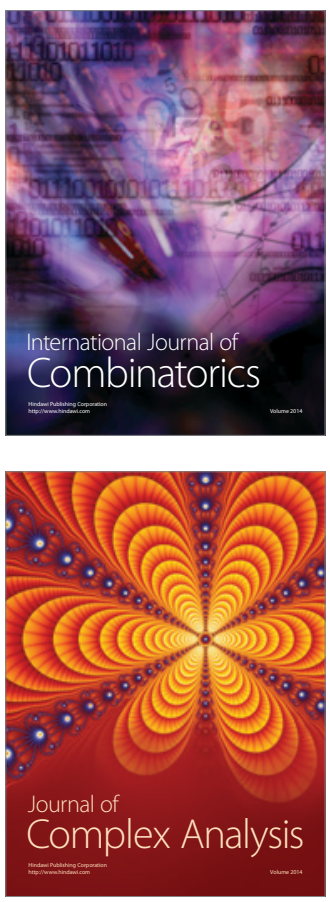

International Journal of

Mathematics and

Mathematical

Sciences
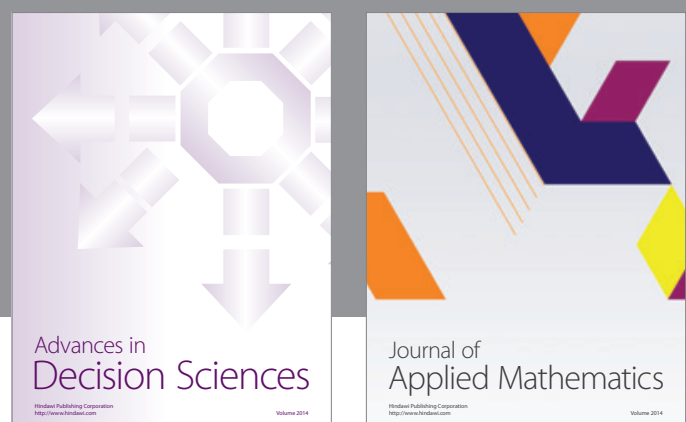

Journal of

Applied Mathematics
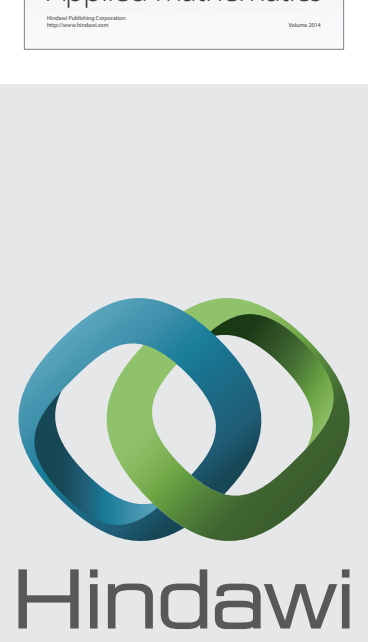

Submit your manuscripts at http://www.hindawi.com
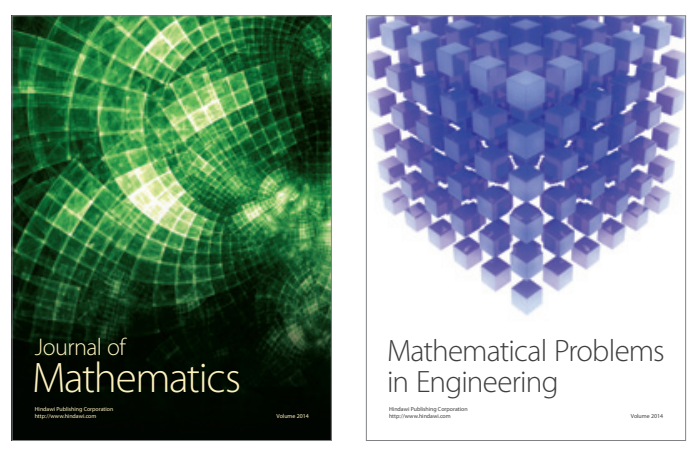

Mathematical Problems in Engineering
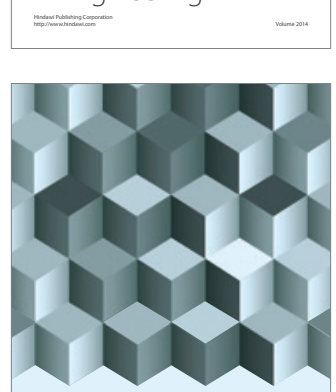

Journal of

Function Spaces
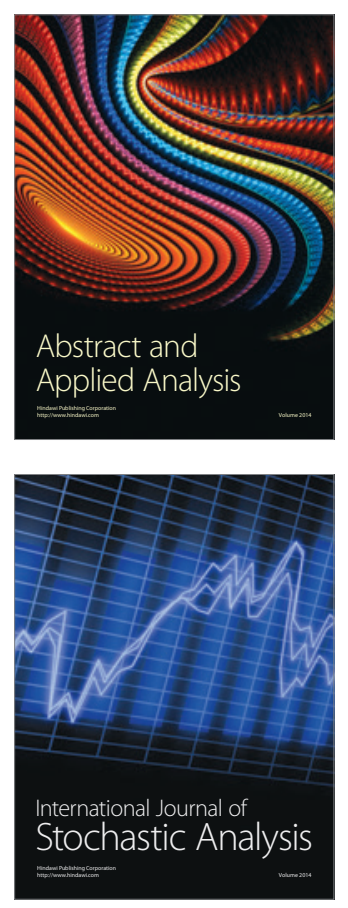

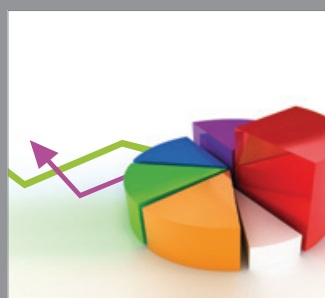

ournal of

Probability and Statistics

Promensencen
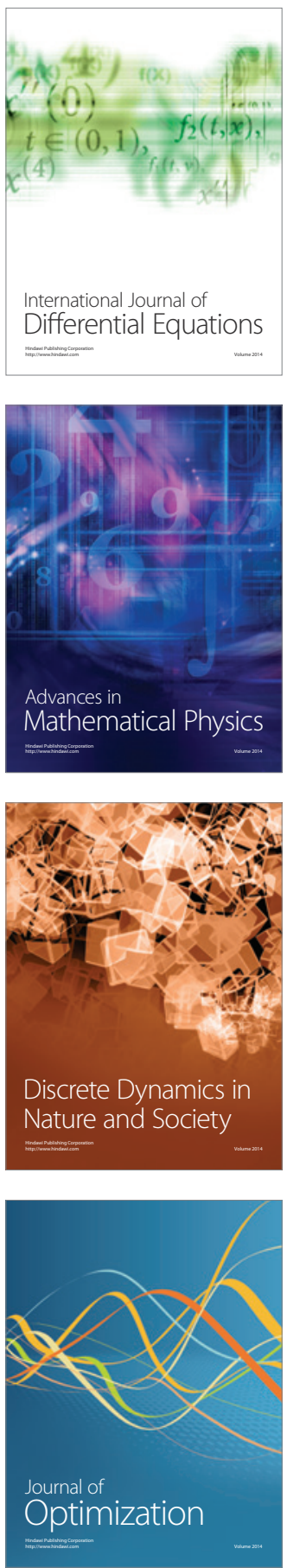